\begin{tabular}{|c|c|c|}
\hline BENTHAM OPEN & $\begin{array}{c}\text { The Open Chemical Engineering } \\
\text { Journal }\end{array}$ & $\begin{array}{l}\text { The open } \\
\text { Chemical nnginering } \\
\text { lournal }\end{array}$ \\
\hline CrossMark & $\begin{array}{l}\text { Content list available at: www.benthamopen.com/TOCENGJ/ } \\
\text { DOI: } 10.2174 / 1874123101711010053\end{array}$ & 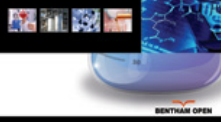 \\
\hline
\end{tabular}

RESEARCH ARTICLE

\title{
Determination of Migration of Six Phthalate Esters in Plastic Toys by GC-MS/MS Coupled with Solid-Phase Extraction Using Cucurbit[n]urils as Adsorbent
}

\author{
Qing Lv, Haiyu Li, Zhijuan Wang, Hua Bai and Qing Zhang* \\ Institute of Industrial and Consumer Product Safety, Chinese Academy of Inspection and Quarantine, Beijing 100176, \\ P.R. China
}

Received: August 01, 2017

Revised: September 13, 2017

Accepted: October 20, 2017

\begin{abstract}
:
Object:

This paper presents a method for the determination of migration of six phthalate esters in plastic toys based on gas chromatographytandem mass spectrometry (GC-MS/MS) coupled with solid phase extraction using cucurbiturils (CB6-8) as adsorbent.

\section{Method:}

On the base of self-made migration device, toy samples were migrated for $10 \mathrm{~min}$ to $24 \mathrm{~h}$ in simulated saliva at $37^{\circ} \mathrm{C}$. The analytes were adsorbed by cucurbiturils SPE cartridges, eluted with ethyl acetate, then determined by GC-MS/MS and quantified by external standard method. The eluting solvents as well as the type and amount of the extraction materials were carefully optimized, then the reusability of the SPE cartridges was investigated. The limits of quantification (LOQs) ranged from $2.5 \mu \mathrm{g} / \mathrm{L}$ to $12.5 \mu \mathrm{g} / \mathrm{L}$. Average recoveries of target analytes (spiked at three concentration levels) were in the range of $82.7 \%$ to $94.4 \%$. Intraday repeatability and interday repeatability of the method varied from $2.3 \%$ to $6.6 \%$ and from $5.6 \%$ to $10.8 \%$, respectively.
\end{abstract}

\section{Conclusion:}

The proposed method was finally used to explore the migration behavior of phthalate esters in commercial plastic toys.

Keywords: Cucurbit[n]uril, GC-MS/MS, Solid phase extraction, Migration, Phthalate esters.

\section{INTRODUCTION}

Cucurbit[n]urils $(\mathrm{CB}[\mathrm{n}]$, where $\mathrm{n}=5,6,7,8,10 ;$ Fig. 1) are pumpkin-shaped macrocyclic host molecules with a hydrophobic cavity and two identical openings lined with carbonyl groups [1]. Given this special structure, $\mathrm{CB}[\mathrm{n}]$ has become a research hotspot in the supermolecule field in recent years. Their interaction with target molecules is mainly driven by (1) the hydrophobicity cage effect, (2) the hydrophilia double-end-open effect, and (3) the size match of cavity effect $[2,3]$. Considering features such as low toxicity, high thermal and chemical stability, as well as potential application in separation science, $\mathrm{CB}[\mathrm{n}] \mathrm{s}$ have been successfully used as the stationary phase of gas chromatography [4 - 7]. Jiang et al., 2011 [8] applied CB6 in solid-phase microextraction as a new coating material to analyze the volatile constituents of amomum cardamomum, but no report on cucurbituril application in solid-phase extraction exists. The unique structure of cucurbiturils enables them to be potential hydrophilic and lipophilic materials in solid-phase extraction.

\footnotetext{
* Address correspondence to this author at the Institute of Industrial and Consumer Product Safety, Chinese Academy of Inspection and Quarantine, Beijing 100176, P.R. China; Tel: +8653897460; E-mails: njuzhangqing@163.com, caiq2011@126.com
} 


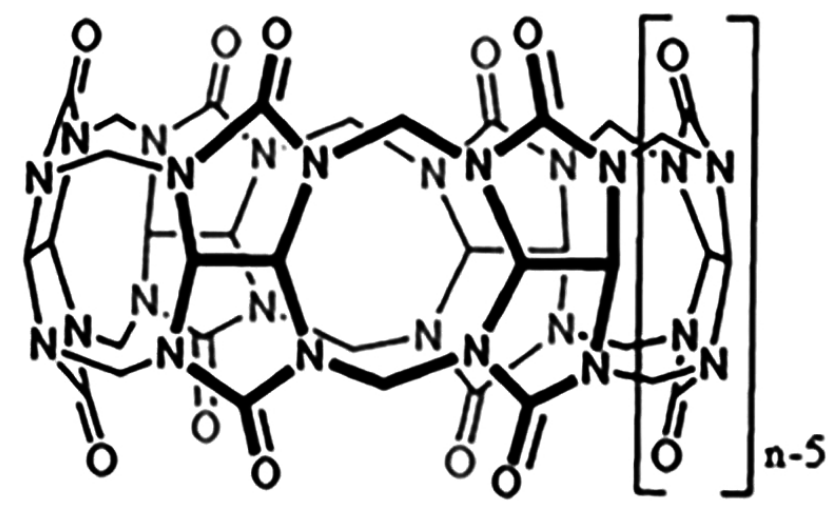

Fig. (1). Structure of cucurbit[n]uril.

Phthalate esters (PAEs) plasticizers have been widely used in numerous products, including toys, food packaging, leather, nail polish, and liquid shampoo, among others. Plasticizers constitute a type of endocrine interfering substances. These materials present toxicity in reproduction, embryo, and heredity. Moreover, these substances can damage immune and reproductive systems and may cause cancer and teratogenesis $[9,10]$. Plasticizers in the plastic toys may migrate into the body through saliva during play, thereby resulting in damage to health. The New Order of Toys of EU 2009/48/E [11] lists six types of plasticizers, including diallyl phthalate (DBP), as restricted substances and restrict total plasticizer content to less than $0.1 \%$. Numerous reports have presented plasticizer detection methods and migration experiments [12 - 14]. However, most of these studies involve products, such as food contact material, and several have mentioned the migration of plasticizers in toys along with saliva. In this article, we attempt to construct a novel solidphase extraction cartridge by using the cucurbituril materials. Important parameters, such as elution solvent, material type and dosage, and SPE cartridge reuse are optimized. In addition, we initially study the migration of six plasticizers in plastic toys through the self-made migration device based on highly sensitive GC-MS/MS technique. The study on migration provides valuable data for exposure assessment, which plays an important role in the formulation of chemical limit standards and supervision measures to promote the safety of toys.

\section{EXPERIMENTAL}

\subsection{Reagents and Materials}

GC-MS/MS analysis was performed using a Varian 450-GC coupled to a Varian 240 ion trap mass spectrometer equipped with a Varian CP-8400 auto-sampler. NTS-4000 thermostatic water bath oscillator (Eyela, Japan); solid-phase extraction device (Supelco, US); 6 mL SPE blank glass cartridge (Dikma, China); SPE manual installation device for column and polyethylene sieve plate with bore diameter of $5 \mu \mathrm{m}$ (Doudian, China).

Diisobutyl phthalate (DIBP), Dibutyl phthalate (DBP), Di-n-pentyl phthalate (DPP), Benzyl butyl phthalate (BBP), Di(2-ethylhexyl) phthalate (DEHP), Di-n-octyl phthalate (DNOP) with purities $>98 \%$ were all purchased from Dr. Ehrenstorfer. Cucurbituril CB6 with purity $>98 \%$ was purchased from Hubei Jusheng Company in China. CB7 and CB8 were synthesized [1], and the products are confirmed through nuclear magnetism (HNMR and CNMR) and high resolution mass spectrum. Acetone, methanol, dichloromethane, ethyl acetate, and hexane were of chromatographic grade (J.T. Baker, USA). Deionized water was obtained from a Milli-Q water purification system (Millipore, USA).

\subsection{Preparation of Standard Solution}

Exactly $100 \mathrm{mg}$ of each standard substance of the six plasticizers was accurately weighed and respectively placed in $100 \mathrm{~mL}$ brown glass flasks. A constant volume was obtained by using ethyl acetate, and a single standard stock solution at $1000 \mathrm{mg} / \mathrm{L}$ was prepared. By using the single standard stock solution, a completely mixed stock solution with concentration of $100 \mathrm{mg} / \mathrm{L}$ was prepared and stored at $4{ }^{\circ} \mathrm{C}$. If applicable in the experiment, the solution was diluted with ethyl acetate to prepare a series of working solutions with the required concentration.

To prepare simulated saliva, we have referred to the standard of DIN53160 of EU [15]. Simulated saliva: $4.5 \mathrm{~g}$ sodium chloride, $0.3 \mathrm{~g}$ potassium chloride, $0.3 \mathrm{~g}$ sodium sulfate, $0.4 \mathrm{~g}$ ammonium chloride, $0.3 \mathrm{~g}$ of $90 \%$ lactic acid, and $0.2 \mathrm{~g}$ urea. A final volume of $1000 \mathrm{~mL}$ was prepared by using deionized water. 
Solution A: A $50 \mu \mathrm{L}$ volume of the completely mixed standard solution containing the six plasticizers at a concentration of $50 \mathrm{mg} / \mathrm{L}$ concentration was added with $20 \mathrm{~mL}$ water and completely mixed.

\subsection{Preparation of SPE Cartridge of Cucurbituril}

$100 \mathrm{mg}$ of cucurbituril was accurately weighed and placed in a $6 \mathrm{~mL}$ blank glass cartridge. The top and bottom were fixed with a matched polyethylene sieve plate. The thickness of material should be distributed uniformly. The material was compacted by a manual pushrod for cartridge installation.

\subsection{Sample Preparation}

The smooth side of plastic toy was obtained, and round pieces with $12 \mathrm{~mm}$ diameter and $1 \mathrm{~mm}$ thickness were cut from the section by a round slicer, with a pore in the center. Five round piece samples were strung together in a line with stainless steel wire, with glass beads in the middle to separate the samples. The samples were placed in a $40 \mathrm{~mL}$ customized colorimetric tube. About $20 \mu \mathrm{L}$ of simulated saliva was added into the tube, which was subsequently subjected to vibration in a water bath at $37{ }^{\circ} \mathrm{C}$ at $100 \mathrm{r} / \mathrm{min}$ for $10 \mathrm{~min}$ to $24 \mathrm{~h}$ to obtain the migration solution. In advance, the migration solution was subjected to prewashing with $5 \mathrm{~mL}$ of methanol, and the cucurbituril SPE column was balanced by $5 \mathrm{~mL}$ of water. After water removal by vacuum pump, the column was eluted with $5 \mathrm{~mL}$ ethyl acetate. Then, the eluent was collected and added with appropriate amounts of anhydrous sodium sulfate for drying and machine testing.

\subsection{Analytical Conditions}

Separations were performed on a DB-5 capillary column $(30 \mathrm{~m} \times 0.25 \mathrm{~mm}$ i.d., $0.25 \mu \mathrm{m})$ from Agilent Technologies. Helium (purity $>99.999 \%$ ) was used as a carrier gas at $1.0 \mathrm{~mL} / \mathrm{min}$. The injector was operated in splitless mode at 280 ${ }^{\circ} \mathrm{C}$, and the inject volume was $1 \mu \mathrm{L}$. The $\mathrm{GC}$ oven temperature was programmed from $80{ }^{\circ} \mathrm{C}$ to $210{ }^{\circ} \mathrm{C}$ at $20{ }^{\circ} \mathrm{C} / \mathrm{min}$, then to $215^{\circ} \mathrm{C}$ at $10{ }^{\circ} \mathrm{C} / \mathrm{min}$, and then to $300{ }^{\circ} \mathrm{C}$ at $30^{\circ} \mathrm{C} / \mathrm{min}$ (held for $5 \mathrm{~min}$ ). A solvent delay of $7 \mathrm{~min}$ was established in order to increase filament's service life. The analytes were ionized by electron ionization (EI), $70 \mathrm{eV}$ in positive ion mode. The EI-MS/MS process was carried out by collision-induced dissociation (CID) using a resonant waveform type.

\section{RESULTS AND DISCUSSION}

\subsection{Optimization of Analytical Condition}

Through optimization of gas chromatography and tandem mass spectrometry technology conditions, the six plasticizers reached good baseline separation within $12 \mathrm{~min}$. The limit of quantitation (LOQ) in this method is less than $12.5 \mu \mathrm{g} / \mathrm{L}$. Chromatographic retention time, monitored ion pair, and linearity range of each substance are shown in Table 1. Fig. (2) indicates the typical chromatogram of the six analytes.

Table 1. Retention time, target ion pair, LOQ, and linear range of the six PAEs under consideration.

\begin{tabular}{|c|c|c|c|c|c|c|}
\hline Compounds & CAS & Retention time (min) & $\begin{array}{c}\text { Target ion pairs (m/z) } \\
\text { and excitation voltage }(\mathbf{V})\end{array}$ & $\begin{array}{c}\text { LOQ }(\boldsymbol{\mu g} / \mathbf{L}) \\
\text { Linear ranges }(\boldsymbol{\mu g} / \mathbf{L})\end{array}$ & $\begin{array}{c}\text { Correlation } \\
\text { coefficients }\end{array}$ \\
\hline DIBP & $84-69-5$ & 8.143 & $\begin{array}{c}149.1>121.2(1.0)^{*} \\
223.1>149.1(1.5)\end{array}$ & 2.5 & $2.5-1250$ \\
\hline DBP & $84-74-2$ & 8.578 & $\begin{array}{c}149.1>121.2(1.0)^{*} \\
223.1>149.1(2.1)\end{array}$ & 2.5 & $2.5-1250$ \\
\hline DNPP & $131-18-0$ & 9.342 & $\begin{array}{c}149.1>121.2(1.0)^{*} \\
237.1>149.1(1.3)\end{array}$ & 2.5 & $2.5-1250$ \\
\hline BBP & $85-68-7$ & 10.069 & $\begin{array}{c}149.1>121.2(0.8)^{*} \\
206.0>149.1(1.0)\end{array}$ & 5 & $5-1250$ \\
\hline DEHP & $117-81-7$ & 10.692 & $\begin{array}{c}149.1>121.2(0.8)^{*} \\
167.0>149.1(1.0)\end{array}$ & 5 & 0.9998 \\
\hline DNOP & $117-84-0$ & 11.537 & $\begin{array}{c}149.1>121.2(0.8)^{*} \\
279.1>149.1(1.2)\end{array}$ & 12.5 & $5-1250$ \\
\hline
\end{tabular}

* Precursor $>$ quantifier (excitation voltage) 


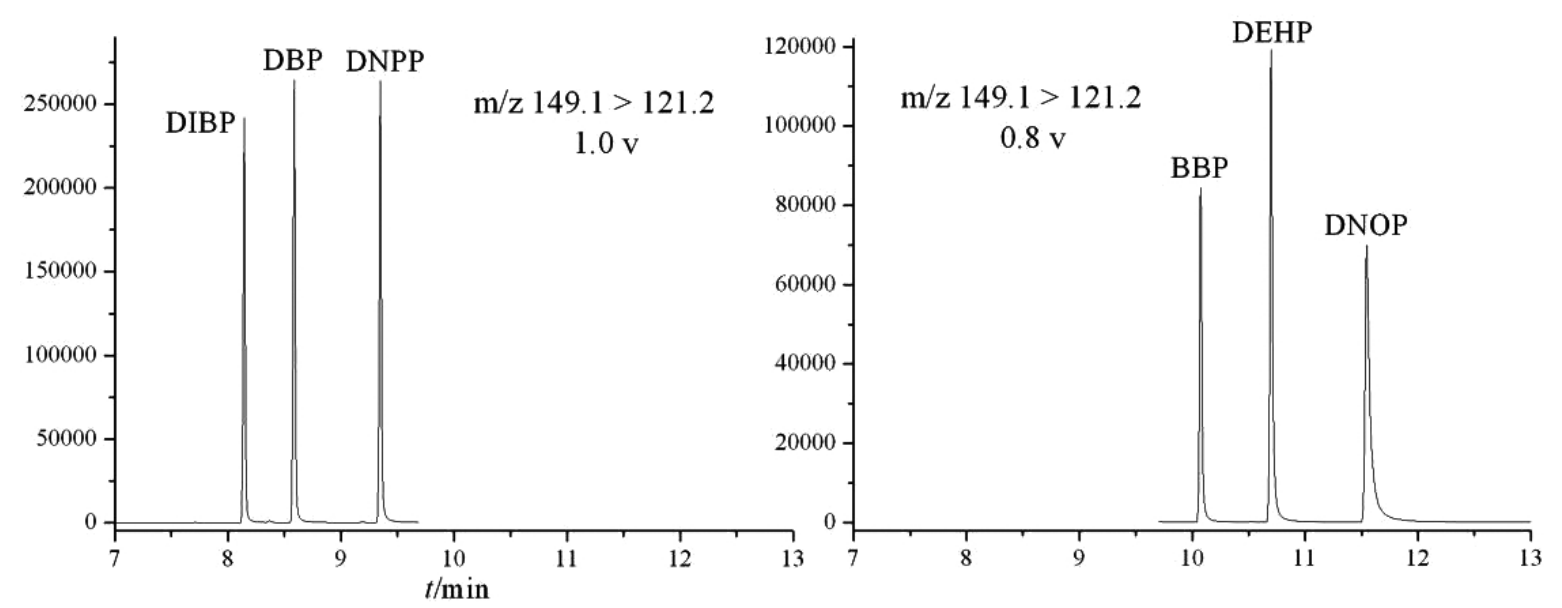

Fig. (2). MRM chromatograms of six PAEs standard solution (2 mg/L).

\subsection{Optimization of Solid-Phase Extraction Conditions}

\subsubsection{Selection of Elution Solvent}

By taking recovery rate as indicator, the elution effects of six plasticizers on CB6 SPE cartridges (100 mg loading) by normal hexane, ethyl acetate, acetone, and dichloromethane have been observed. Solution A (see preparation method in Section 2.2) is passed through prewashing by $5 \mathrm{~mL}$ methanol, and the CB6 cartridge is balanced by $5 \mathrm{~mL}$ water in advance. After taking water out by vacuum pump, the cartridge is respectively eluted with $5 \mathrm{~mL}$ each of the four abovementioned solvents.

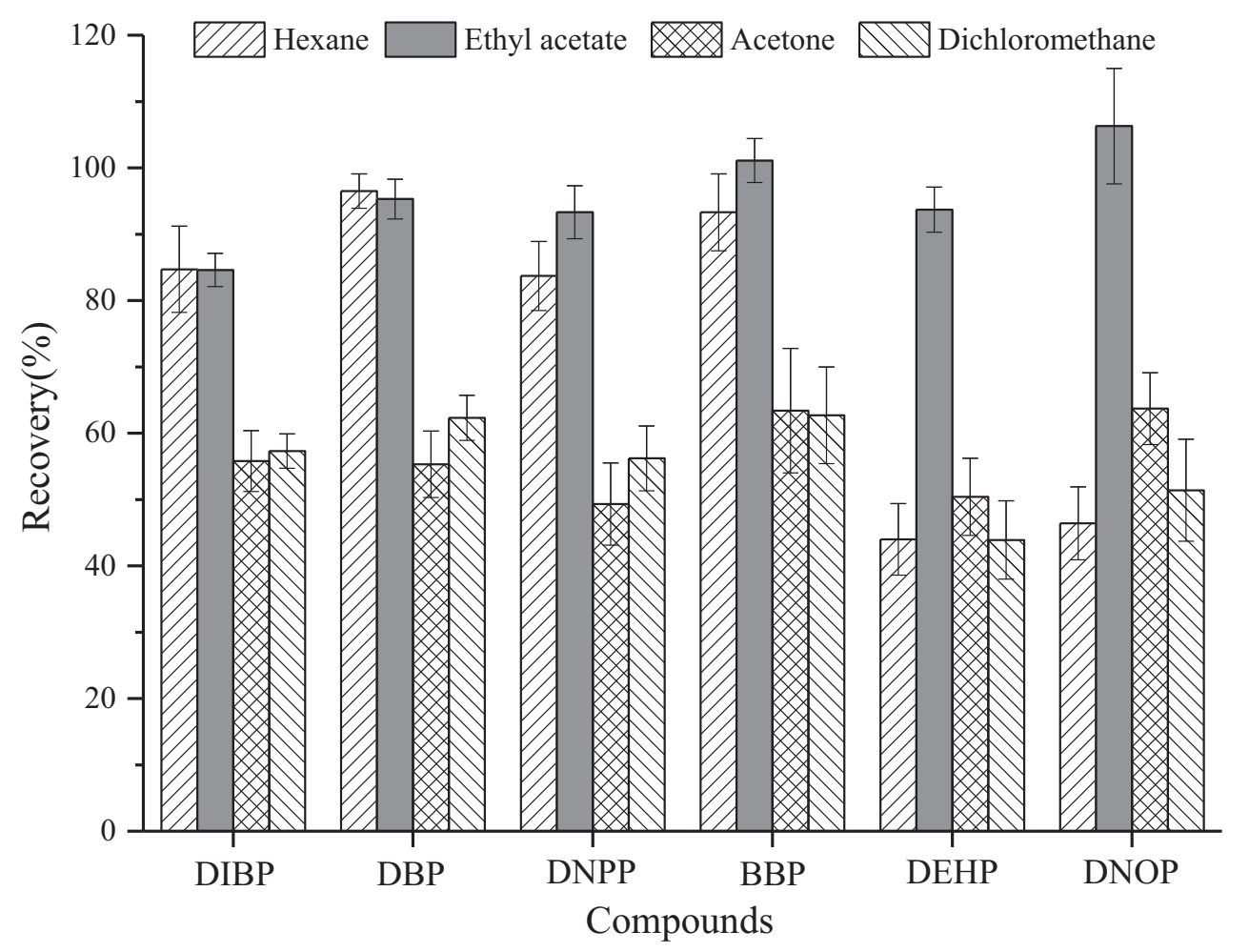

Fig. (3). Comparison of six PAEs elution effects with different solvents ( $\mathrm{n}=5)$. 
Obtained recovery rates are indicated in Fig. (3). Results show significant differences among the elution effects by four solvents. Acetone and dichloromethane show the poorest effects (with all recovery rates of six substances less than $65 \%$ ). Hexane presents good elution effects on DIBP, DBP, DNPP, and BBP, but its DEHP and DNOP recovery rates are less than 50\%. Ethyl acetate as elution solvent yields the highest recovery rates (84\% to $106 \%)$ and better repeatability $(\mathrm{RSD} \leq 8.4 \%)$. Probably because ethyl acetate and phthalate esters both contain ester group, ethyl acetate gives the highest recovery rates based on the principle of similar dissolution. Thus, $5 \mathrm{~mL}$ ethyl acetate is selected as the elution solvent in this method.

\subsubsection{Optimization of Extraction Material Type and Dosage}

The filling amount of extraction materials in SPE cartridges exerts certain influence on experimental results. Insufficient dosage cannot completely adsorb all target substances, whereas excessive dosages will affect sample loading speed and elution efficiency. For SPE cartridges of three types of cucurbiturils, namely, CB6, CB7 and CB8, we observe through the experiment the extraction effect under filling amount of 50, 100, and $150 \mathrm{mg}$, respectively. Solution A is passed through SPE cucurbituril cartridges with different filling amount and eluted by $5 \mathrm{~mL}$ ethyl acetate. The obtained recovery rate of each substance tested in triplicate $(\mathrm{RSD}<10 \%)$ is shown in Fig. (4). Results indicate that, within $50 \mathrm{mg}$ to $150 \mathrm{mg}$, the recovery rate of each substance generally increases with the increase of filling amount. Given the special structure, the size of the benzene ring on benzyl group of BBP $(\approx 5.8 \AA)$ approaches that of the cucurbituril CB7 cavity (opening diameter: $5.4 \AA$ and inner diameter: $7.4 \AA$ ) [16]. Thus the ring could enter the cavity, resulting in strong adsorption. Several filling amounts can achieve good recovery rate, but $50 \mathrm{mg}$ filling cannot completely adsorb other substances, especially DEHP and DNOP (recovery rate less than 60\%). In terms of filler types, CB6, CB7 and CB8 exert highly similar extraction effects on the six types of plasticizer. At the filling amounts of 100 and $150 \mathrm{mg}$, recovery rates through the extraction of CB6 and CB8 both exceed that of CB7, and the recovery rate of each substance all surpasses $80 \%$. Thus, we finally select the CB6 cartridge with filling amount of 100 mg to measure plasticizer migration amount.

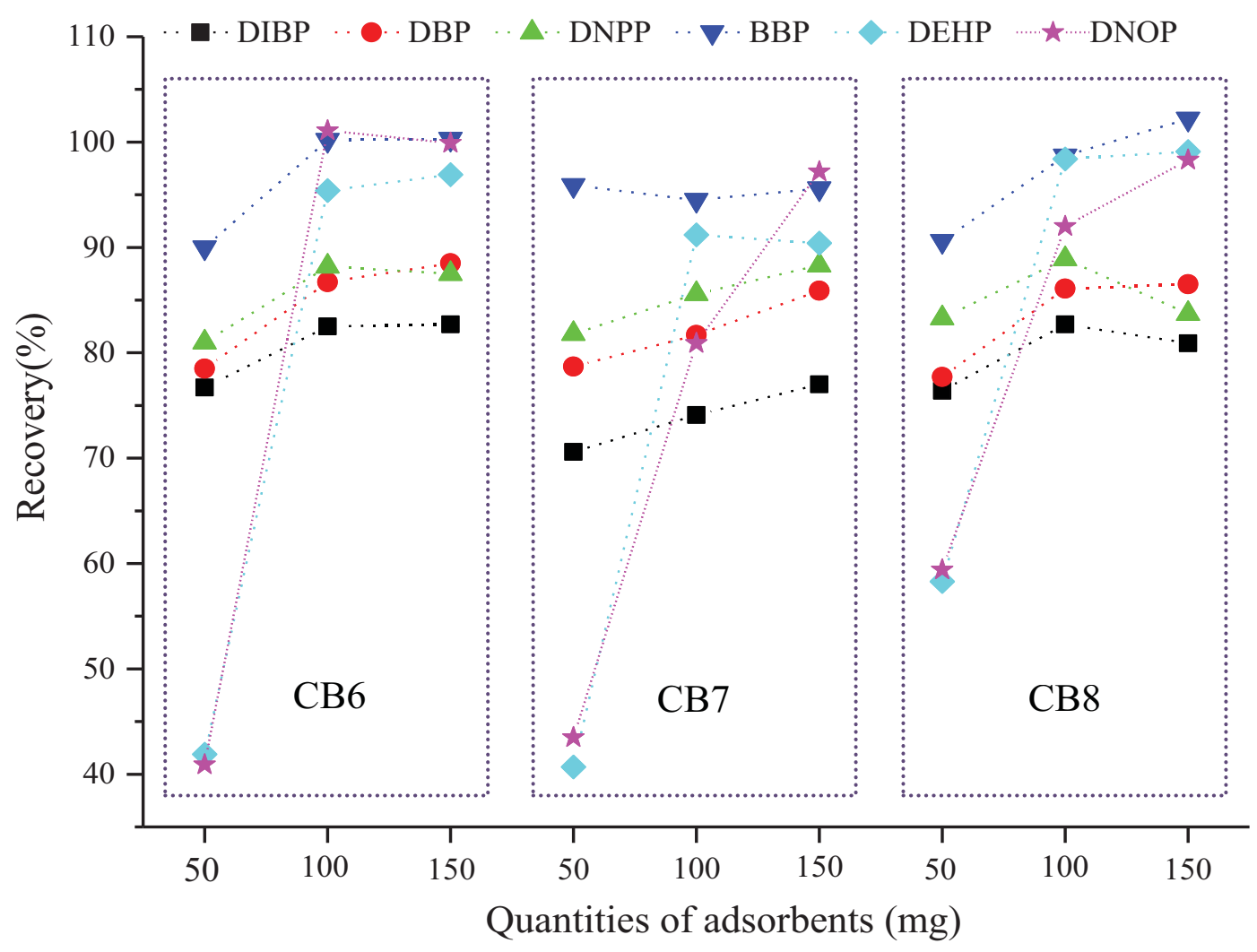

Fig. (4). Comparison of extraction effects of six PAEs with different amounts of three adsorbents( $\mathrm{n}=3)$. 


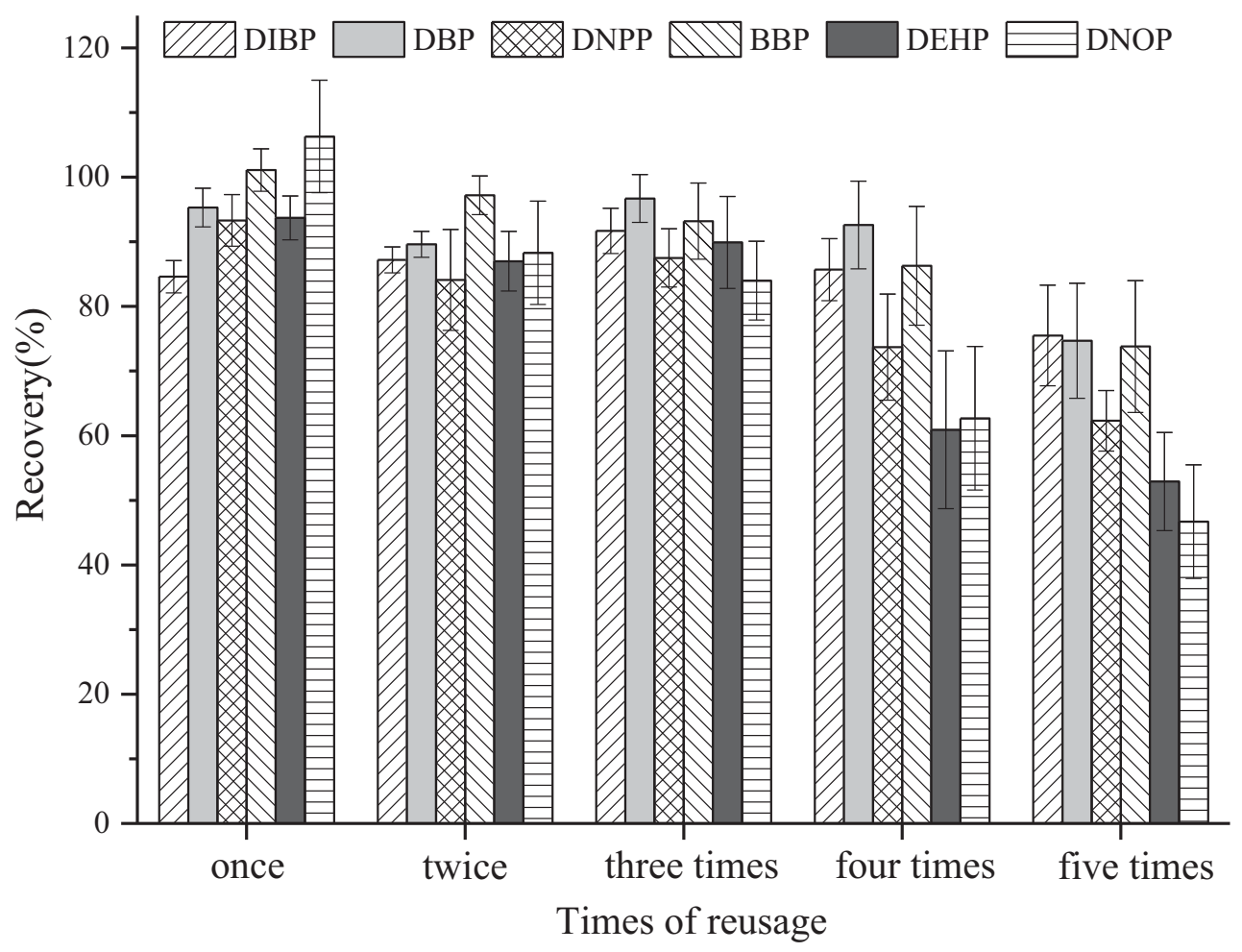

Fig. (5). Comparison of six PAEs recoveries when CB6 SPE cartridge are reused five times ( $\mathrm{n}=5)$.

\subsubsection{Observation of SPE Cartridge Reuse}

Manufacturers of SPE cartridges will generally stress that the product is disposable. In practice, however, people prefer SPE cartridges to be used for several times to save costs. The reuse of SPE cartridges strongly depends on the complexity of sample matrix solution. With a highly complicated matrix solution, the possibility of reuse of SPE cartridges is smaller. Through the experiment, we observe CB6 SPE cartridge recycling through repetitive operations (prewashed by $5 \mathrm{~mL}$ methanol $\rightarrow$ balanced by $5 \mathrm{~mL}$ water $\rightarrow$ passed through solution $\mathrm{A} \rightarrow$ eluted by $5 \mathrm{~mL}$ ethyl acetate). We can see from Fig. (5) that the CB6 cartridge can guarantee that the recovery rate of each substance is higher than $80 \%$ after three reusage times. Then, the recovery rate starts to sharply decrease after the 4 th use.

\subsection{Recovery Rate and Repeatability}

Three different concentration levels of each plasticizer (namely, low, medium, and high, are set) are added in $20 \mathrm{~mL}$ simulated saliva, and extracted by CB6 cartridge with a filling amount of $100 \mathrm{mg}$. On the basis of the confirmed experimental conditions in this method, we conduct six experiments at each added concentration. The obtained recovery rate of each substance is from $82.7 \%$ to $94.4 \%$. The relative standard deviation of repeatability within a day (RSD, $\mathrm{n}=$ 6) ranges from $2.3 \%$ to $6.6 \%$, and the relative standard deviation of repeatability (RSD, $n=4$ ) among days ranges from $5.6 \%$ to $10.8 \%$. Detailed data are indicated in Table 2 .

Table 2. Recoveries, intraday and interday repeatabilities of six PAEs.

\begin{tabular}{|c|c|c|c|c|c|}
\hline \multirow{2}{*}{ Compounds } & \multirow{2}{*}{ LOQ $(\boldsymbol{\mu g} / \mathbf{L})$} & \multicolumn{2}{|c|}{$\begin{array}{c}\text { Recoveries (\%) and intraday repeatabilities } \\
\text { (RSD, } \mathbf{n = 6 , \% )}\end{array}$} & $\begin{array}{c}\text { Interday repeatabilities } \\
\text { (RSD, } \mathbf{n}=\mathbf{4}, \mathbf{\%})\end{array}$ \\
\cline { 3 - 6 } & & low concentration & $\mathbf{1 2 5} \boldsymbol{\mu g} / \mathbf{L}$ & $\mathbf{2 5 0} \boldsymbol{\mu g} / \mathbf{L}$ & $\mathbf{1 2 5} \boldsymbol{\mu \mathbf { g } / \mathbf { L }}$ \\
\hline DIBP & 2.5 & $82.7(2.3)$ & $89.9(4.7)$ & $85.3(5.3)$ & 9.4 \\
\hline DBP & 2.5 & $88.5(5.5)$ & $90.2(3.6)$ & $87.8(2.3)$ & 10.8 \\
\hline DNPP & 2.5 & $88.0(5.3)$ & $91.1(2.7)$ & $91.1(3.3)$ & 5.6 \\
\hline BBP & 5 & $88.8(6.6)$ & $94.4(3.4)$ & $92.1(2.7)$ & 8.6 \\
\hline DEHP & 5 & $88.7(5.1)$ & $87.6(3.8)$ & $91.3(4.4)$ & 7.9 \\
\hline DNOP & 12.5 & $85.2(3.6)$ & $89.1(5.4)$ & $87.2(2.6)$ & 7.7 \\
\hline
\end{tabular}

Note: Added concentration at a low concentration of each substance: $5,5,5,5,5$, and $12.5 \mu \mathrm{g} / \mathrm{L}$. 


\subsection{Determination of Migration Amount of Plasticizer}

The self-made migration device is illustrated in Fig. (6). We obtained the smooth side of a plastic toy and cut round pieces with diameter of $12 \mathrm{~mm}$ and thickness of $1 \mathrm{~mm}$ by a round slicer, with a pore in the center. The five round sample pieces were strung in a line with stainless steel wire, and glass beads placed between each sample for separation. The line was placed in the $40 \mathrm{~mL}$ customized colorimetric tube. A $20 \mathrm{~mL}$ volume of simulated saliva into the tube, which was then placed in a water bath at $37^{\circ} \mathrm{C}$ at $100 \mathrm{r} / \mathrm{min}$ for $10 \mathrm{~min}, 30 \mathrm{~min}, 1 \mathrm{~h}, 5 \mathrm{~h}$, and $24 \mathrm{~h}$ for vibration, so as to obtain the migration solution. The migration solution was then extracted in CB6 SPE cartridge and eluted by ethyl acetate for test.

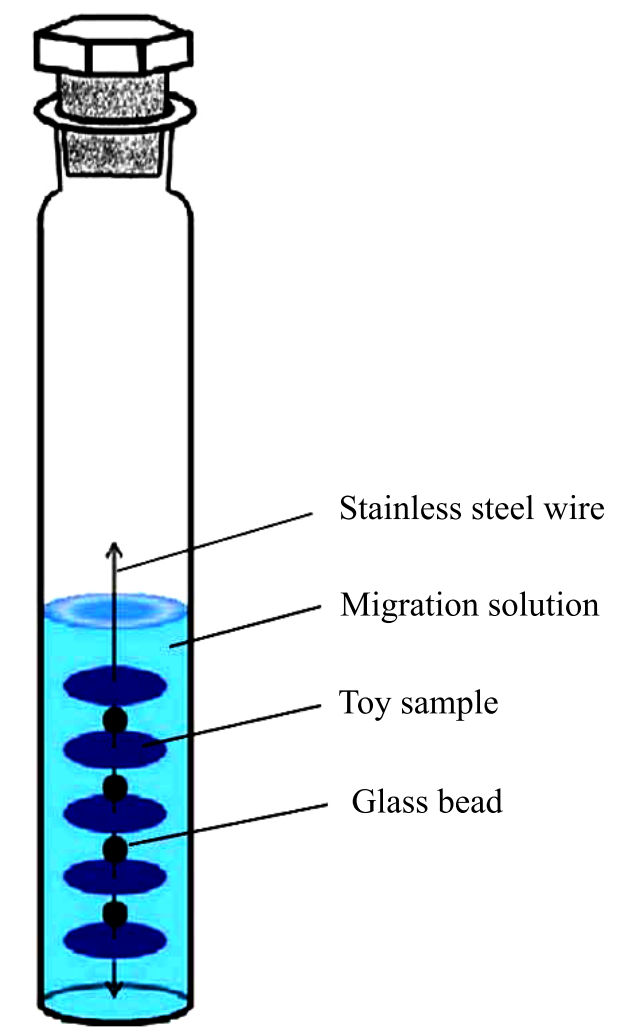

Fig. (6). Schematic diagram of self-made migration device.

This method is applicable for plastic toys of all textures. We tested 14 plastic toy samples that were randomly collected in the market by this method. The textures of the toys included PP, ABS, and PS, but those of other toys were not marked. Results indicate that each toy sample contains a minimum of two types of plasticizers, and five types of toys contain four types of plasticizers. We detected a total of four types of plasticizers, namely, DIBP, DBP, DNPP, and DEHP. DIBP, DBP and DEHP are found in almost all 14 toy types. Thus, we can infer that these plasticizers are more commonly used in the production of plastic toys.

We analyzed the migration of DIBP, DBP, and DEHP by selecting six types of toys with high amounts of the analytes. We can see from Fig. (7) that the migration laws of different plasticizers in each toy sample vary with the increase of migration time when each point is tested in triplicate ( $\operatorname{RSDs}<10 \%$ ). The migration law is related to the initial substance. For the same sample, for example, DIBP in sample 4, the migration amount will gradually increase with the increase of migration time, whereas the migration volumes of DBP and DEHP are almost stable or even decrease with the increase in migration time. This trend may be caused by the balance between the toy and migration medium within short time. The migration amount is also related with the texture of sample. For the same substance, such as DIBP for example, the migration amounts in samples 1, 2, 4, and 5 gradually increase with the increase in migration time (and the growth trend is different). In contrast, migration in samples 3 and 6 is balanced after a short period. This finding indicates that a considerable quantity of plasticizer has migrated into the body, posing health hazard despite the short time when children place these toys in their mouths. 

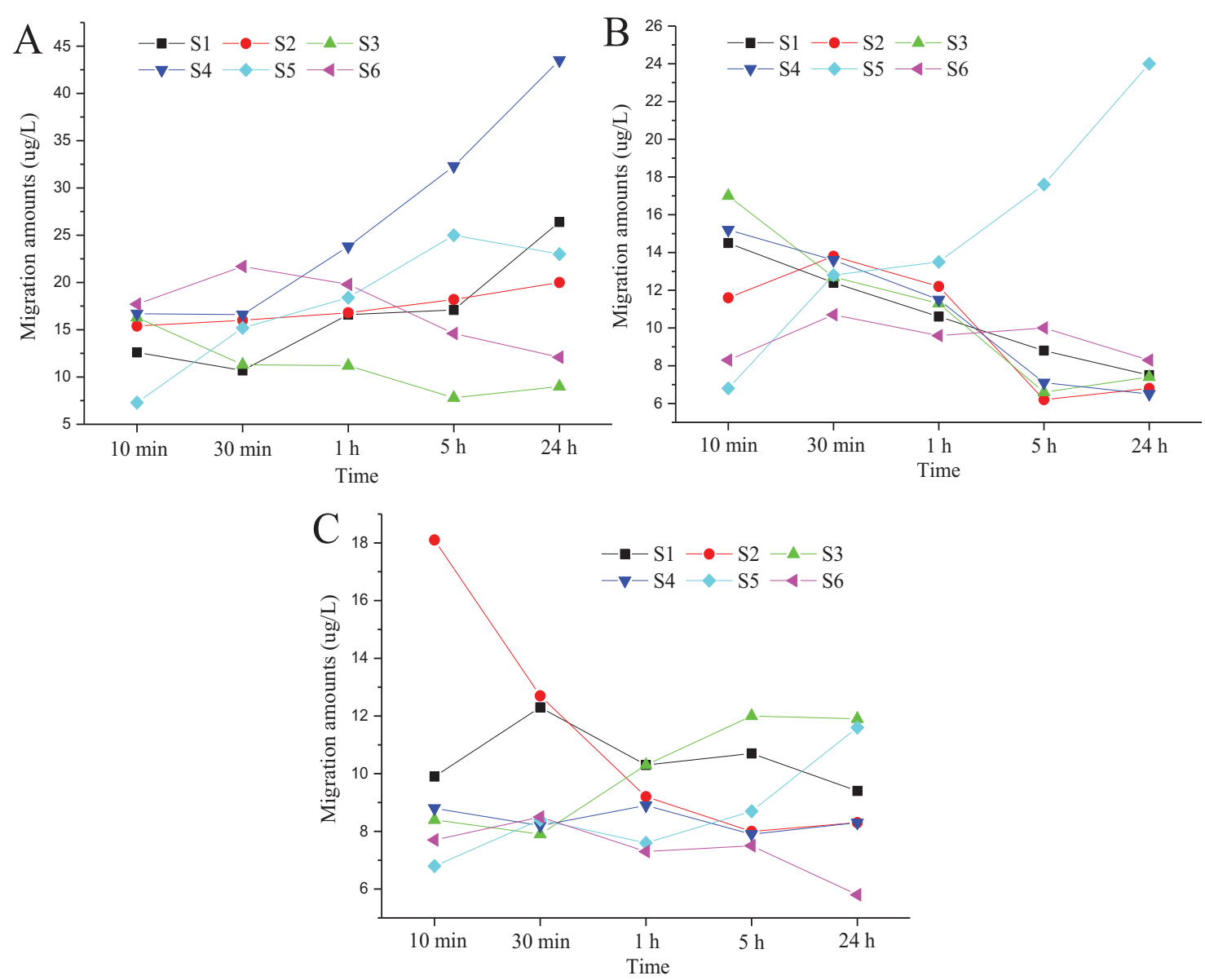

Fig. (7). Migration results of three PAEs in simulated saliva at $37^{\circ} \mathrm{C}(\mathrm{n}=3)$. A: DIBP; B: DBP; C: DEHP

See Fig. (8) for chromatogram of blank matrix (practical samples not including plasticizers in the experiment of migration) and chromatogram of sample 4 after $24 \mathrm{~h}$ through migration.
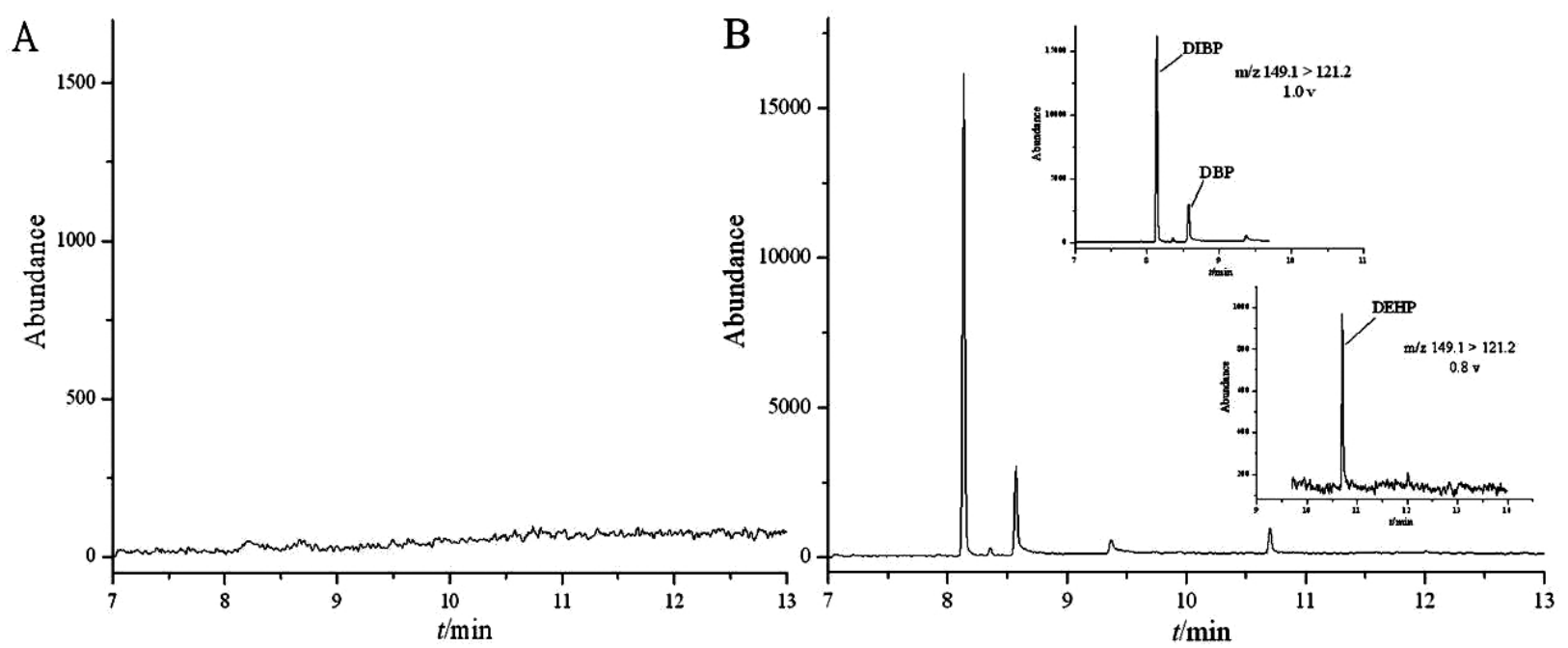

Fig. (8). A. MRM chromatogram of blank sample; B. MRM chromatogram of sample 4 migrated for $24 \mathrm{~h}$. 


\section{CONCLUSION}

For the first time, cucurbiturils are used for the extraction of plasticizers in aqueous solution by acting as solid-phase extraction material in this article. A method of measuring the migration amount of six types of plasticizers in plastic toys is established on the basis of gas chromatography and tandem mass spectrum analysis. By testing the samples of practical plastic toys, the migration behavior of plasticizers in toys is analyzed. Results indicate that cucurbiturils can be effectively used in solid-phase extraction, and their potential needs further exploration. The established method is accurate, sensitive, stable, and can be used for practically detecting the migration amount of plasticizer in plastic toys.

\section{CONSENT FOR PUBLICATION}

Not applicable.

\section{CONFLICT OF INTEREST}

The authors declare no conflict of interest, financial or otherwise.

\section{ACKNOWLEDGEMENTS}

This work was financially supported by the Science Research Program of the Chinese Academy of Inspection and Quarantine (No.2014JK017) and the Quality Inspection Public Welfare Scientific Research Foundation (No.201310057) .

\section{REFERENCES}

[1] J. Kim, I.S. Jung, S.Y. Kim, E. Lee, J.K. Kang, S. Sakamoto, K. Yamaguchi, and K. Kim, "New cucurbituril homologues: syntheses, isolation, characterization, and X-ray crystal structures of cucurbit[n]uril ( $\mathrm{n}=5,7$, and 8)", J. Am. Chem. Soc., vol. 122, pp. 540-541, 2000. [http://dx.doi.org/10.1021/ja993376p]

[2] D. Bardelang, K.A. Udachin, D.M. Leek, J.C. Margeson, G. Chan, C.I. Ratcliffe, and J.A. Ripmeester, "Cucurbit[n]urils (n = 5-8): a comprehensive solid state study", Cryst. Growth Des., vol. 11, pp. 5598-5614, 2011. [http://dx.doi.org/10.1021/cg201173j]

[3] E. Masson, X. Ling, R. Joseph, L. Kyeremeh-Mensah, and X. Lu, "Cucurbituril chemistry: a tale of supramolecular success", RSC Advances, vol. 2, pp. 1213-1247, 2012. [http://dx.doi.org/10.1039/C1RA00768H]

[4] P. Zhang, S. Qin, M. Qi, and R. Fu, "Cucurbit[n]urils as a new class of stationary phases for gas chromatographic separations", $J$. Chromatogr. A, vol. 1334, pp. 139-148, 2014. [http://dx.doi.org/10.1016/j.chroma.2014.01.083] [PMID: 24565233]

[5] L.S. Li, S.W. Wang, X.Q. Chen, C. Liu, and L.L. Xu, "Studies on chromatographic properties of perhydroxycucurbit[6]uril as a new type of gas chromatographic column packing material", Chin. J. Chem., vol. 26, pp. 307-314, 2008. [http://dx.doi.org/10.1002/cjoc.200890060]

[6] L. Wang, X. Wang, M. Qi, and R. Fu, "Cucurbit[6]uril in combination with guanidinium ionic liquid as a new type of stationary phase for capillary gas chromatography", J. Chromatogr. A, vol. 1334, pp. 112-117, 2014.

[http://dx.doi.org/10.1016/j.chroma.2014.01.070] [PMID: 24560922]

[7] T. Sun, M. Qi, and R. Fu, "Perhydroxylcucurbit[6]uril as a highly selective gas chromatographic stationary phase for analytes of wide-ranging polarity", J. Sep. Sci., vol. 38, no. 5, pp. 821-824, 2015. [http://dx.doi.org/10.1002/jssc.201400876] [PMID: 25529611]

[8] Y.L. Jiang, M.L. Qi, and R.N. Fu, "Cucurbiturils as a new coating materials for solid phase microextraction", Chem. J. Chin. Univ., vol. 32, no. 9, pp. 2215-2218, 2011.

[9] L.Z. Huang, X.X. Zhang, T.G. Zhu, and X.L. Zhang, "The direct analysis of diethyl phthalate in perfume by mass spectrometry", Journal of Chinese Mass Spectrometry Society, vol. 35, no. 6, pp. 550-554, 2014.

[10] F. Zhang, Z. Li, Y. Zhang, Z. Huang, and X. Wang, "Determination of six phthalate acid esters in camellia oil by gas chromatography-mass spectrometry coupled with solid-phase extraction using single-walled carbon nanotubes as adsorbent", $\mathrm{Se} P u$, vol. 32, no. 7, pp. 735-740, 2014.

[http://dx.doi.org/10.3724/SP.J.1123.2014.04036] [PMID: 25255566]

[11] F. Zhang, Z. Li, Y. Zhang, Z. Huang, and X. Wang, "Determination of six phthalate acid esters in camellia oil by gas chromatography-mass spectrometry coupled with solid-phase extraction using single-walled carbon nanotubes as adsorbent", $\mathrm{Se} P u$, vol. 32, no. 7, pp. 735-740, 2014. [http://dx.doi.org/10.3724/SP.J.1123.2014.04036] [PMID: 25255566]

[12] H.J. Zhang, X.J. Hu, and S.B. Lin, "Determination of 15 phthalate esters in drinking water by solid phase extraction-ultra performance liquid chromatography-tandem mass spectrometry", Chin. J. Anal. Chem., vol. 42, no. 9, pp. 1281-1287, 2014. 
[13] X. Zheng, L. Lin, E. Fang, Y. Huang, S. Zhou, Y. Zhou, X. Zheng, and D. Xu, "Determination of 23 phthalate esters in food by solid-phase extraction coupled with gas chromatography-mass spectrometry", Se Pu, vol. 30, no. 1, pp. 27-32, 2012. [http://dx.doi.org/10.3724/SP.J.1123.2011.08019] [PMID: 22667087]

[14] J.X. Song, Z.H. Yang, and L.Q. Chen, "Progress on determination and migration of phthalate esters plasticizer in foods", Chemical Analysis and Meterage, vol. 22, no. 1, pp. 100-102, 2013.

[15] J.X. Song, Z.H. Yang, and L.Q. Chen, "Progress on determination and migration of phthalate esters plasticizer in foods", Chemical Analysis and Meterage, vol. 22, no. 1, pp. 100-102, 2013.

[16] M. Buaki-Sogo, P. Montes-Navajas, M. Alvaro, and H. Garcia, "Host-guest complexes between cucurbit[n]urils and acetanilides having aminopropyl units", J. Colloid Interface Sci., vol. 399, pp. 54-61, 2013. [http://dx.doi.org/10.1016/j.jcis.2013.02.027] [PMID: 23541695]

\section{(C) 2017 Lv et al.}

This is an open access article distributed under the terms of the Creative Commons Attribution 4.0 International Public License (CC-BY 4.0), a copy of which is available at: https://creativecommons.org/licenses/by/4.0/legalcode. This license permits unrestricted use, distribution, and reproduction in any medium, provided the original author and source are credited. 